\title{
DIE \\ GEFÄSSVERSORGUNG DER SEHNEN
}

\author{
VON \\ ERICH RAU, \\ LEIPZI G.
}

(DIE ARBEIT WURDE UNTER LEITUNG VON PROF. DR. SPALTEHOLZ IM ANATOMISCHEN INSTITUT DER UNIVERSITAT LEIPZIG AUSGEFUHRT.)

Mit 12 Abbildungen auf den Tafeln $38 / 41$. 
Die Blutgefässversorgung der Sehnen ist wiederholt Gegenstand von Untersuchungen gewesen, und verschiedene Methoden sind benutzt worden, um einen klaren Einblick in die Verhältnisse zu gewinnen. Aber immer noch bestehen Zweifel darüber, ob überhaupt im Innern der Sehnen Blutgefässe vorhanden sind. Deshals, beauftragte mich Herr Prof. Dr. Spalteholz, die Frage von der Gefässversorgung der Sehnen an der Hand von Injektionspräparaten mit der von ihm ausgearbeiteten Aufhellungsmethode erneut zu prüfen.

Ehe ich die von mir angewandte Technik beschreibe, will ich in kurzen Zügen die bisherigen Angaben in der Literatur wiedergeben, soweit sie für den Gang meiner Arbeit in Betracht kommen.

Koelliker ${ }^{1}$ ) sagt: „Die Sehnen gehören zu den an Blutgefässen ärmsten Teilen des Körpers. Kleinere Sehnen sind im Innern ohne alle Spur von Blutgefässen, besitzen dagegen äusserlich in dem mehr lockeren Bindegewebe, das sie umbüllt, reichliche weitmaschige Capillarnetze. Bei stärkeren Sehnen finden sich auch in den oberflächlichen Sehnenlagen einzelne Gefässchen und bei den stärksten lassen sich durch Mikroskop und Einspritzungen spärliche Gefässnetze auch in tieferen Schichten nachweisen; doch sind auch hier die innersten Sehnenteile vollkommen gefässlos. - Wie die Sehnen ver-

1) Handbuch der Gewebelehre. 2. Aufl. 1855. S. 199. (Wörtlich über. nommen in die 6. Aufl. 1889. Bd. 1. S. 381.) 
halten sich auch die Bänder der Sehnen, nar dass in ihnen noch weniger Gefässe nachzuweisen sind."

1887 steilte $\mathrm{Berkenbusch}{ }^{1}$ ) Untersuchungen an über die Blutzufuhr zu den Beugesehnen der Hände und Füsse innerhalb der Ligg. intravaginalia. Durch Injektion mit wässeriger Lösung von Berliner Blau gelang es ihm, nachzuweisen, dass die Sehnen mit Gefässen versehen sind, welche von der Insertionsstelle am Knochen, von der Palmarfläche her und durch die Vincula tendinum herantreten. Die meisten dieser Gefässe, besonders die aus den Vincula tendinum stammenden, bleiben auf der Oberfläche der Sehnen, eine Anzahl geht jedoch in die Substanz hinein. Trotzdem sind an den Sehnen Stellen in einer Ausdehnung von etwa $1 \mathrm{~cm}$ ohne Injektion. Als Material verwandte Berkenbus ch Hände und Füsse erwachsener Menschen und aus Mangel an kindlichem menschlichen Material Sehner von jungen Tieren. Er fand dabei, dass bei jungen Schweinen und Kälbern die Sehnen reichlicher mit Blutgefässen versorgt sind als bei erwachsenen Menschen. Indem er nun einfach diesen Befund am Tier auf den Menschen übertrug, kam er zu der Annahme, dass beim Menschen die in der Jugend vorhandenen Gefässe im Laufe des Wachstums teilweise obliterieren, und stellte die Vermutung auf, dass die Versorgung der volaren Fingersehnen abhängig ist von der Grösse der Sehnen und vom Aiter ihres Trägers.

Eine Arbeit von Tichonow ${ }^{2}$ ) aus dem Jahre 1902, die mir im Original nicht zugänglich war, scheint nur eine sehr ausführliche Beschreibung der an die Sehnen herantretenden

') Die Blutversorgung der Beugesehnen der Finger. Nachrichten der Kgl. Gesellschaft d. Wissensch. Göttingen 1887. S. a. Merkel, Handbuch der topographischen Anatomie des Menschen. 3. Bd. S. 628, 629.

2) Die Blutgefässe der langen Sehnen an der volaren Flache des Vorderarmes und der Hand. Russk. chir. Arch. Bd. 18. 1902. S. 850-874 (Russisch). Referiert in: Jahresber. über d. Fortschr. d. Anat. u. Entwicklungsgesch. Hernusgeg. v. G. Sehwalbe. N. F. Bd. IX (f. 1908). 3. Teil. S. B11. 
Gefässe, aber nichts über ihre Verteilung im Innern derselben zu enthalten.

Ausgedehntere Versuche über die Sehnenarterien wurden später von Wollenberg ${ }^{1}$ ) mit Hilfe der Röntgenstrahlen angestellt. Er injizierte seine Präparate (von Kinderm und Erwachsenen) mit einer 50\%igen Quecksilberterpentinemulsion und befreite dann die Sehnen von dem sie umhüllenden Bindegewebe (Peritenonium externum), um auf der Röntgenplatte möglichst nur die in der Sehne selbst verlaufenden arteriellen Gefässe zu Gesicht zu bringen. Er kommt bei seiner Arbeit zu dem Ergebnis, dass ,weder beim Erwachsenen noch beim Neugeborenen Arterien nachzuweisen sind, welche in der Längsrichțung der Sehnen verlaufen", gibt jedoch zu, dass von den in der Peripherie der Sehne liegenden Gefässen feine Ästchen in das Peritenonium internum, das die einzelnen Sehnenfaserbündel trennt, hineinziehen, im wesentlichen in radiärer Richtung.

Dieses in der Hauptsache negative Resultat Wollenbergs ist in der angewandten Methodik begründet. Erstens ist eine 50\%ige Quecksilberterpentinemulsion für eine feine Gefässinjektion zu dickflüssig. Zweitens aber ist die Röntgentechnik zum Nachweis von Gefässen nur in beschränktem Masse anwendbar, insofern als feinere injizierte Gefässe durch die Röntgenstrahlen überhaupt nicht sichtbar gemacht werden. Darauf hat zuerst $\mathrm{Spal}$ te $\mathrm{holz}{ }^{2}$ ) aufmerksam gemacht, nachdem es ihm nicht gelungen war, deutlich an der Oberfläche des Herzens sichtbare Anastomosen mit Röntgenstrahlen phiotographisch zu fixıeren.

1) Die Arterienversorgung von Muskeln und Selunen. Zeitschr. für orthopäd. Chirurgie. Bd. 14. 1905.

2) Die Coronararterien des Herzens. Verhandlungen der anatom. Gesellsch. auf der 21. Versamml. in Würzburg 1907. Anatom. Anz. Suppl. 1907. S. 145. 
Zur selben Zeit, aber unabhängig von Wolle nberg, hat Araic) an Sehnen Injektionsversuche mit einer wässerigen Lösung von japanischer Tusche angestellt. Nach der Injektion fixierte er seine Präparate in 10\%igem Formol und fertigte von ihnen mikroskopische Schnitte von $15-20 \mu$ Dicke, sowie Totalpräparate an. Die Abbildungen sind Autotypien nach photographischen Aufnahmen. Die Ergebnisse seiner Untersuchungen über den Verlauf der Blutgefässe der Sehnen lassen sich, wie folgt, zusammenfassen:

„a) Die Arterien der Sehnen kommen 1. aus Zweigen von Muskelarterien" (d. h. zum Muskel verlaufenden Arterien. $\mathrm{R} \mathrm{au}$ ) ,und aus den die Sehnen umgebenden Binde- und Fettgewebsarterien; 2. aus den Arterien des Gelenkes; 3. aus den durch die Vincula tendinum gehenden Arterien; 4. aus den im Muskelfleisch liegenden Arterien und 5. aus den Arterien der Knochenhaut und der Bänder.

b) Die Verästelungsverhältnisse der Arterien der Sehnen variieren; ebenso hat die Form der Anastomosen der Zweige und der Capillarnetze der Sehnenarterien verschiedenen Charakter, je nachdem dieselben an Sehnenabschnitte gehen, welche 1. der Schleimscheiden entbehren, 2. mit Schleimscheiden versehen sind oder 3. an Ansatzstellen der Sehnen liegen.

c) An einzeInen Stellen fehlen Blutgefässe bei den untersuchten Objekten. In die blutgefässlosen Stellen sind Knorpelzellen eingelagert.

d) Die Venae comitantes der Sehnen finden sich vorwiegend einfach, zum kleineren Teil doppelt."

Arai ist also ganz im Gegensatz zu Wollenberg zu einem positiven Resultat gekommen, und seine Bilder zeigen sogar einen grossen Reichtum von Blutgefässen! Kein Wunder! Denr er injizierte mit einer Flüssigkeit, welche ausser den

') Die Blutgefüsse der Sehnen. Anatom. Hefte. Bd. 34 (1907). S. 363. 
Arterien auch die Capillaren und Venen füllte. Abgesehen von dem weiteren oder weniger weiten Vordringen der Massen in den Arterien, lassen sich die Bilder Wollenbergs und A rais deshalb gar nicht miteinander vergleichen; bei letzteren müssen wir die gefüllten Capillaren und Venen, also mehr als die Hälfte der Blutgefässe, abziehen, um eine Vorstellung von der Menge der. vorhandenen Arterien zu erhalten. Von besonderer Wichtigkeit ist es, dass die Präparate A ra is fast ausnahmslos von Neugeborenen gewonnen wurden. Unter 24 Abbildungen zeigt nur Figur 19 (Längsschnitt durch die Sehne des M. peronaeus longus) die Gefässanordnung in der Sehne beim „Erwachsenen“. A rai erwähnt auch nichts von einem Unterschied in der Gefässanordnung zwischen den Sehnen jugendlicher und erwachsener Personen; cr überträgt einfach seine Befunde beim Neugeborenen auf das höhere Alter.

Bei der Betrachtung der früher angestellten Versuche und der damit erzielten Resultate mussten sich für mich ohne weiteres zwei Wege ergeben, die ich bei meinen Untersuchungen einzuschlagen hatte: erstens musste ich bei der Injektion meiner Objekte eine Injektionsmasse verwenden, die auch in die feinerer Arterien eindringen kann, die Capillaren aber nicht füllt; zweitens war es notwendig, Sehnen von Neugeborenen und von Erwachsenen zum Gegenstand meiner Untersuchungen zu machen.

Ich injizierte nach genügendem Vorwärmen und nach Durchspülen mit warmer physiologischer Kochsalzlösung obere und untere Extremitäten von Kindern und Erwachsenen mit einer auf $38-40^{\circ} \mathrm{C}$ angewärmten Masse aus zwei Teilen $15 \%$. iger Gelatinelösung und einem Teil feinsten Zinnobers. Dieser Masse setzte ich geringe Mengen von löslichem Berliner Blau zu, um für das spätere Photographieren der Präparate möglichsi günstige Verhältnisse zu gewinnen; das reine Zinnober hat nur sehr wenig aktinische Strahlen; der Zusatz von Blau ver- 
grössert ihre Menge wesentlich. Die in 10\%igem Formalin fixierten Sehnen schnitt ich' grob' heraus, präparierte sie dann sauber frei vom Peritenonium externum und behandelte sie weiter nach der Aufhellungsmethode von Spalteholz'): Die Präparate wurden in Wasserstoffsuperoxyd gebleicht, dann in steigenidem Alkohol entwässert und nach dreimaligem Aufenthalt in Benzol schliesslich in das Endgemisch (5 Gewichtsteile Wintergrünöl unid 3 Gewichtsteile Benzylbenzoat) gebracht. Die Abbildungen sind nach unretouchierten Originalphotographien meiner Präparate hergestellt.

Obwohl ich die Achilles- und die Quadricepssehne zum Hauptgegenstand meiner Untersuchungen gemacht habe, unterlasse ich es doch nicht, auch einige Photographien von anderen Sehnen zu bringen. Ich habe den einzelnen Figuren, die ja den Hauptbestandteil meiner Arbeit bilden sollen, Erläuterungen beigegeben, so dass ich mich mit der Beschreibung der einzelnen Präparate hier kurz fassen kann. Die Reihenfolge ist eine derartige, dass die Abbildungen (mit Ausnahme von Fig. 4, Taf. 2) nach dem Alter des Trägers der Sehnen zusammengestellt sind.

Bei der Schilderung meiner Befunde beginne ich zunächst mit den Sehnen des Neugeborenen. Figur 1 stellt die Achillessehne eines neugeborenen Kindes dar; sie zeigt im wesentlichen dieselbe Anordnung der Gefässe, die auch A rai bei seinen Injektionspräparaten von Neugeborenen gefunden und beschrieben hat. Man sieht innerhalb der Sehne Arterien in der Längsrichtung verlaufen und miteinander anastomosieren. Teilweise entspringen sie aus dem im Muskel gelegenen Arteriennetz, durchziehen dann die Sehne der Länge nach und bilden am Knochenansatz feine Schlingen; teilweise stehen sie auch am Rande des Knochenansatzes mit Arterien in Verbindung, welche vom Periost, aus in den Knochen eindringen, so dass die Periost-

1) Uber das Durchsichtigmachen von menschlichen und tierischen Präparaten. Leipzig 1911. S. 39. 
arterien eine indirekte Verbindung zwischen den Sehnen- and den Knochengefässen herstellen. Arterien, welche aus ider Sehne direkt in den Knochen eindringen, habe ich, ebenso wie A rai, nicht finden können. Andere Arterien gelangen vom Peritenonium externum her in die Sehnensubstanz und teilen sich dort dichotomisch in proximale und distale Zweige, und diese geben wiederum Ästchen ab, welche die Sehne senkrecht zur Längsrichtung durchsetzen. Auch bilden die vom Peritenonium aus eindringenden Äste Anastomosen mit den Arterien, welche den Muskelgefässen entstammen. Diese Blatgefässversorgung der Achillessehne durch die im Peritenonium externum gelegenen Arterien erfolgt ausschliesslich von der der Cutis abgewandten Seite her.

Die gleichen. Verhältnisse finden wir an der Quadricepssehne des Neugeborenen (Fig. 3). Auch hier nehmen wir im Innern längsverlaufende Gefässe wahr; doch stammen diese alle aus Arterien, welche vom Peritenonium aus senkrecht in die Sehne eindringen. Ein direkter Übergang von Sehnenarterien in die Patella oder Tibia ist nicht vorhanden. Vielmehr endigen die feineren Arterien im Knochenende der Sehne in feinen Schlingen.

Die Abbildung der Achillessehne eines dreijährigen Knaben (Fig. 5) zeigt uns die gleiche Gefässanordnung. Die Zahl der im Innern längsverlaufenden Arterien, sowie der aus dem Peritenonium externum eintretenden Gefässe ịst eine so grosse, dass wir die Blutgefässversorgung der Sehne als eine reichliche bezeichnen können. Ebenso enthält die Sehne des M. flexor carpi radialis eines dreijährigen Knaben (Fig. 7) längsverlaufende Gefässe, die sich verästeln und miteinander anastomosieren.

Die Sehnen eines 17 jährigen Mädchens (Fig. 8) und eines 23 jährigen Mannes (Fig. 10) unterscheiden sich bemerkenswerterweise weder im ganzen Aufbau des Gefässnetzes noch in dessen Einzelteilen von denen des 3 jührigen Knaben. Ich 
möchte darauf besonders hinweisen, da keiner der früheren Untersucher einen derartigen Gefässreichtum der Sehnen bei erwachsenen Menschen beschrieben hat.

Ganz andere Verhältnisse zeigen dagegen die Achillessehnen bei älteren Individuen. Schon bei einem Mann von 28-29 Jahren ist die Menge der im Innern der Sehne längs verlaufenden Gefässe ausserordentlich reduziert; ganz fehlen diese Gefässe aber bei einem 39 jährigen (Fig. 11) und einem 45 jährigen Manne (Fig. 12). Wohl besitzt bei diesen Sehnen das Peritenonium externum grössere Gefässe; welche, wie die Abbildungen zeigen, perforierende Ästchen abgeben; diese dringen aber nur auf sehr kurze Strecken in die Sehnensub$\operatorname{stanz}$ ein und bilden dort keinerlei odor nur unbedeutende Anastomosen. Die Muskelgefässe setzen sich im Gegensatz zu den Verhältnissen, wie wir sie bei jugendlichen Personen angetroffen haben, nicht in das Innere der Sehne fort, sondern treten auf deren Oberfläche über.

Eine kurze Zusammenfassung der Ergebnisse meiner Injektionsversuche ergibt folgendes: Der Blutgefässreichtum im Innern der Sehnen ist bei Neugeborenen und Kindern ein ausserordentlich grosser. Auch bei Erwachsenen, die das Alter von ca. 25 Jahren noch nicht erreicht haben, sind noch zahlreiche Arterien im Innern der Sehnen vorhanden, während bei Individuen oberhalb dieser Grenze eine deutliche Gefässverminderung zu erkennen ist. Es lassen sich also zu den oben angeführten, von A rai aufgestellten Sätzen noch zwei weitere hinzufügen :

I. Die Sehnen bei Neugeborenen, Kindern und Erwachsenen bis ungefähr zum 25. Lebensjahre haben im allgemeinen eine doppelte Blutgefässversorgung, eine innere von Arterien aus, welche im Innern der Sehne in der Längsrichtung verlaufen, und eine äussere von den Gefässnetzen des Peritenonium ex- 
ternum aus; beide geben rechtwinklig Ästchen ab, welche miteinander anastomosieren können.

II. Bei Personen über ca. 30 Jahren erhält die Sehne nur wenige kurze perforierende Blutgefässäste von dem sie umhüllenden Bindegewebie (Peritenonium externum), während das Innere der Sehne gefässarm ist.

Die Zahl der Präparate, welche ich für die Entscheidung der angeschnittenen Frage herangezogen habe, ist mit den im Text erwähnten und abgebildeten durchaus nicht erschöpft, denu ich hatte noch oft die Möglichkeit, injizierte Sehnen untersucher zu können. Ich habe die Gefässverhältnisse bei jeder Gelegenheit mit der binocularen Lupe bzw. mit dem binocularen Mikroskop sorgfältig studiert. Alle Fälle aber stimmen in den geschilderten Verhältnissen genau überein und fügen sich ein in diə beiden neu aufgestellten Sätze. Aus begreiflichen Gründen konnte ich jedoch die Zahl der Abbildungen nicht weiter vermehren.

Die Tatsache, dass beim Erwachsenen eine so starke Reduktion der Sehnengefässe eintritt, bestätigt die Hypothese von $\mathrm{B}$ erke $\mathrm{nbus} \mathrm{ch}$ in vollem Masse. Ist es auch sonst nicht ohne Weiteres zulässig, von den Befunden bei jugendlichen Tieren auf diejenigen bei Menschen zu schliessen, so entsprach im vorliegenden Falle dieser Schluss doch den tatsächlichen Verhältnissen.

A rai ist dieser Umstand der Reduktion verborgen geblieben. Seine bereits erwähnte Figur 19, die vom „Erwachsenen" stammt, ist ohne genaue Altersangabe und kommt daher für uns nicht in Betracht; nach der Menge der vorhandenen Gefässe nehme ich an, dass sie von einem ungefähr 25 jährigen Individuum herrührt.

Die Resultate dieser Untersuchungen haben besonderes Interesse sowohl für den Biologen, als auch für den Chirurgen. 
Demi Biologen fallen folgende wichttige Punkte auf. Zunächst ist die Tatsache im hohen Grade interessant, dass die Ernährung der Sehne auf zwei ganz verschiedene Arten möglich ist: einmal in der Jugend durch reichliche Blutgefässe, zweitens nach dem 25. Lebensjahre unter fast vollständigem Ausschluss der Blutgefässe durch Lymphgefässe ${ }^{1}$ ).

Aus dem Umstande, dass man an den Sehnen von Erwachsenen wenig oder gar keine Blutgefässe fand, hat man geschlossen, dass die chemischen Umsetzungen in ihnen sehr gering seien. Dieser Schluss ist nach der Auffindung. des Blutgefüssnetzes bei jugiendlichen Sehnen mindestens für diese nicht mehr zulässig, und vielleicht auch nicht für das spätere Lebensalter. Die Talsache, dass innerhalb der Sehnen, wie Spalteholz²) fand, viel grössere Mengen von Protoplasma vorhanden sind als man bisher in ihnen vermutete, weist ja ebenfalls idarauf hin, dass in der Sehne recht erhebliche chemische Umsetzungen stattfinden, viel erheblichere, als man vielfach geglaubt hat.

Zweitens ist die Frage von besonderer Wichtigkeit, warum es bei den Sehnen überhaupt zu einer Änderung in der Gefässversorgung kommt, und warum dies in dem erwähnten Alter stattfindet. Es lassen sich da zunächst zwei Möglichkeiten mehr physiologischen Charakters denken.

Nehmen wir das eine Mal an, dass die Sehne durch die Lymphgefässe weniger gut versorgt wird als durch die Blutgefässe, so ist es nicht verständlich, inwiefern ihr Ernährungsbedürfnis gerade um die Zeit des 25. Lebensjahres geringer wird. Es entspricht ja dieses Alter dem Abschluss des körperlichen Wachstums; das ist aber wohl nur so zu verstehen, dass

1) Ludwig and Schweigger-Seidel, Die Lymphgefässe der Fascien und Sehnen. Leipzig 1872.

$\left.{ }^{2}\right)$ Verhandlung. d. anatom. Gesellsch. auf d. 20. Vers. in Rostock i. M. 1906. Anatom. Anzeiger Suppl. 1906. S. 215. 
damit die Vorbedingungen für die grösste Entfaltung der vorhandenen Kräfte erfüllt sind, und dass der Körper damit auf einem Höhepunkt anlangt, auf dem er sich zweifellos durch eine Reihe von Jahren erhält. Und mitten in dieser Periode tritt eine Änderung in der Gefässversorgung ein! Wir kennen nichts, was uns veranlasst, anzunehmen, dass die Sehne eines z. B. 23 jährigen Individuums andere biologische und mechanische Leistungen zu erfüllen hat und damit andere chemische Umsetzungen zeigt als die Sehne eines vielleicht 33 jührigen Individuums. Wenn nach dieser Annahme die Ernährung der Sehne am Ende dieser Periode eine ungünstigere wäre als am Anfang derselben, so verstehen wir den Grund dafür nicht, da die Sehne zu beiden Zeitpunkten durchaus die gleichen Bedingungen und die gleichen biologischen und mechanischen Verhältnisse darzubieten scheint.

Wenn aber nach: einer zweiten Annahme die Versorgung der Sehne durch Lymphgefässe der durch Blutgefässe gleichwertig ist, dann ist uns ein physiologischer Grund für eine Änderung erst recht nicht verständlich.

Es widerstrebt uns, diesen Vorgang in eine gewisse Parallele zu setzen mit den Veränderungen, wie wir sie im späteren Lebensalter an anderen. Geweben als Clbergang zum senilen Zustand kennen, zumal da wir für diese Ïnderungen plausible Gründe (Abnutzung durch fortgesetzten Gebrituch) anführen können, während uns solche bei der Sehne zunïchst fehlen. Es scheint widersinnig zu sein, dass an einem Gewebe, das zur vollen Entwickelung kommt und nicht der frühzeitigen Rückbildung unterworfen ist, eine „Alterserscheinung" bereits beginnt, ehe es voll entwickelt ist.

So bleibt uns also nur die Möglichkeit, die Tatsache der Änderung in der Ernährung der Sehne mit ihrer „Entwickelung“" in Verbindung zu bringen. 
Es ist ja zu auffällig, dass die Rückbildung der Blutgefässe ziemlich genau zu der Zeit beginnt, wo das Wachstum des Körpers abgeschlossen ist. Wenn auch, wie bereits ausgeführt, die physiologischen Verhältnisse für die Sehne sich mit dem Abschluss des Wachstums zunächst nicht zu ändern scheinen, so ist es doch denkbar, dass bei der Sehne ebenso wie bei andern Organen die Zeit des Wachsens - und sei dieses auch nur ein langsames - eine grössere Zufuhr bestimmter im Blute zirkulierender Stoffe bedingt, als die spätere Periode. Und weiter lässt sich denken, dass die eben erwähnten Stoffe zugleich einen Reiz'auf die Gefässwand ausüben, der zur Erhaltung der Gefässe notwendig ist. Wenn nach dem Abschluss des Wachsens diese Einflüsse zu wirken aufhören, dann verschwindet damit auch die Ursache für das Offenbleiben der Gefässe, und diese atrophieren allmählich.

Diese Erklärung bewegt sich allerdings vorläufig ganz auf dem Boden der Hypothese, steht aber unter den möglichen Erklärungen am wenigsten im Widerspruch mit den uns geläufigen Tatsachen und Anschauungen und scheint daher zunächst wenigstens als Arbeitshypothese brauchbar.

Weitere Untersuchungen werden hier hoffentlich bald Klarheit bringen.

Den Chirurgen interessieren die Ernährungsverhältnisse besonders wegen der Heilungsvorgänge bei Sehnenwunden (Sehnenplastik, Sehnentransplantation, Tenotomie). Unseren bisherigen Kenntnissen nach scheinen im allgemeinen an gut ernährten, insbesondere gut durchbluteten Geweben die Heilungsvorgänge bessere und günstigere $\mathrm{zu}$ sein als an anderen. Danach müssten also vom theoretischen Standpunkt aus Sehnenaffektionen bei Individuen unter 25 Jahren günstiger ablaufen als bei älteren. Eine genaue Durchsicht der. Literatur hat mir zunächst keine Beweise für diese Vermutung geliefert. Vielleicht ist es nur notwendig, die Aufmerksamkeit auf diesen 
Punkt zu lenken, um manchen rätselhaften Einzelfall zu erklären. Nur bei M e $\mathrm{k} \mathrm{el}^{1}$ ) habe ich eine diesbezügliche Angabe gefunden. Er sagt: „Es ist also die Ernährung der Beugesehnen“ der Finger „eine überaus schlechte, und man versteht, dass sie bei pathologischen Insulten, besonders bei einem Panaritium tendinosum, sehr leicht der Nekrose verfallen müssen, und dass Operationen an ihnen sich durch wenig gute Resultate auszeichnen“. Da Merkel nicht auf das Alter der jeweiligen Patienten hinweist, so spricht diese beilüufige Erwähnung nicht gegen meine Schlussfolgerung. Interessant wäre es aber, durch genaue statistische Aufzeichnungen zu prüfen, ob die klinischen Erfahrungen mit den aus den anatomischen Verhältnissen abgeleiteten Anschauungen übereinstimmen.

1) Handbuch der topographischen Anatomie des Menschen. Bd. 3. S. 628,629 . 


\section{Erklïrung der Abbildungen.}

Sämtliche Abbildungen sind photographiscle Aufnahmen, die ich sclbst von den in dem ölgemisch liegenden Sehnen gemacht habe. An den Pholographien ist keinerlei Retouche irgendwelcher Art vorgenommen worden. Dass einzelne Stellen der Photographien nicht ganz scharf erscheinen, ist in der Dicke der Prüparate begründet; wic ich die Sehnen stets im ganzen, ohne sie in Schnitte zu zerlegen, durchsichtig gemacht habe, so habe ich sie auch unzerschnitten photographiert. Ich bemerke dies ausdrücklich, um den Unterschied meiner Photographien von denen $\Lambda \mathrm{ra}$ is, die teilweise nach dünnen Schnitten angefertigl sind, verständlich zu machen.

An allen Präparaten ist das Peritenonium externum sorgfältig entfernt. Die sichtbaren Gefässe der Sehne lieğen also in ihrem Innern. Sie sind sämtlich Arterien.

Figur 1. Achillessehne eines neugeborenen Knaben, von hinten gesehen. Vergr. 5 fach. Die Abbildung zeigt die doppelte Arterienversorgung der Sehne. Die einen Gefässe kommen vom Muskel her und verlaufen in der Längsrichtung; die anderen kommen rom Peritenonium externum her, dringen senkrecht in die Sehne ein und sind auf der Figur als kurze, dicke Stämmchen zu erkeninen.

Figur 2.. Achillessehne eines neigeborenen Mädchens, von rorn gesehen. Vergr. 5 fach. Die Abbildung zeigt in der Hauptsache die im Innern der Sehne längsverlaufenden Arterien.

Figur 3. Ligamentum patellac eines neugeborenen Mädchens, von vorn gesehen. Vergr. 5 fach. Die Figur zeigt deutlich, dass dic längsverlaufenden Gefässe miteinander anastomosieren und ein reiches Netz von Arterien bilden.

Figur 4. Sehne des M. biceps brachii eines 7 monatigen weiblichen Fetus. Vergr. 40 fach. An dieser Figur sieht man gut die arkadenförmige Anordnung der längsverlaufenden Arterien im Innern der Sehne.

Figur 5. Achillessehne eines 3 jährigen Knaben, von vorn gesehen. Vergr. 4 fach. Die annähernd quer zur Längsachse der Sehne verlaufende Arterie a gibt einesteils direkte Äste in die Knochensubstanz ab und hängt andernteils unmittelbar mit dem Arteriennetz der Sehne zusammen; dadurch bildet sie cine indirekte Anastomose zwischen Schnen- und Knochenarterien. Die mit $h$ und $c$ bezeichneten stärkeren Blutgefässe dringen rom Peritenonium externum her in die Sehnensubstanz ein. 
Figur 6. Ligamentum patellae eines 3 jährigen Knaben, von hinten gesehen. Vergr. 4 fach. Die Abbildung zeigt, dass die Sehnenarterien an der Stelle, wo sich die Sehne an der Tibia anheftet (x), in feinen Schlingen endigen und nicht in die Knochensubstanz eindringen.

Figur 7. Sehne des M. flexor carpi radialis eines 3 jährigen Knaben. Vergr. 5 fach. Die Abbildung zeigt, dass zwar zwischen den einzelnen Gefässversorgungsgebieten ( $a, b, c)$ Anastomosen bestehen, dass aber Bezirke verschiedener Gefässdichtigkeit vorhanden zu sein scheinen. Es ist leicht verständlich, dass bei der Injektion die Anastomosen zwischen den verschiedenen Gebieten sich zuletzt füllen, bei Anwendung ungeeigneter Massen aher überhaupt ungefüllt bleiben können; diese Talsache erklïrt vielleicht die ,gefässlosen" Stellen, die Berken bus ch in der Sehne gefunden hat.

Figur 8. Achillessehne eines 17 jährigen Mädchens, von vorn gesehen. Vergr. 3 fach. Die Abbildung zeigt, dass an der Ansatzstelle der Sehne am Calcaneus im Periost Arterien verlaufen, welche sowohl mit dem Arteriennetz der Schne, als auch mit dem des Knochens zusammenhängen und dadurch indirekte Anastomosen zwischen den Sehnen- and Knochen. arterien bilden. Direkte Verbindungen zwischen den Arterien der Selme und des Knochens sind nirgends sichtbar.

Figur 9. Sehne des M. extensor hallucis longus cines 17 jährigen Mïdchens. Vergr. 5 fach. Die Abbildung zeigt die Herkunft der im Innern der Seline verlaufenden Grefässe: 1. ans Muskelgefässen (a), 2. aus Gefíssen des Peritenonium externum (b).

Fïgur 10. Achillessehne eines 23 jährigen Mannes, von vorn gesehen. Vergr. 3 fach. Die Figur zeigt besonders das Eindringen von Blutgefissen aus dem Peritenonium externum. Dic Sehnenarterien gehen in die Knochensubstanz nicht direkt hinein, sondern endigen in feinen Schlingen $(x)$ oder anastomosieren durch Periostgefässe indirekt mit den Knochenarterien.

Figur 11. Achillessehne eines 39 jährigen Mannes, von vorn gesehen. Vergr. 1:2. Die Abbildung zeigt, wie die im Muskelinnern verlaufenden Arterien sich nicht in das Innere der Sehne fortsetzen, sondem auf deren Oberfläche (Peritenonium externum) treten. In der Sehne selbst sind nur wenige kurze Gefässchen zu sehen.

Figur 12. Achillessehne eines 45 jährigen Mannes, von vorn gesehen. Vergr. 2:3: Die kurzen, im Sehneninnem sichtbaren Arterien sind per. forjerende Ästchen der im Peritenonium externum verlaufenden Gefiissc. 


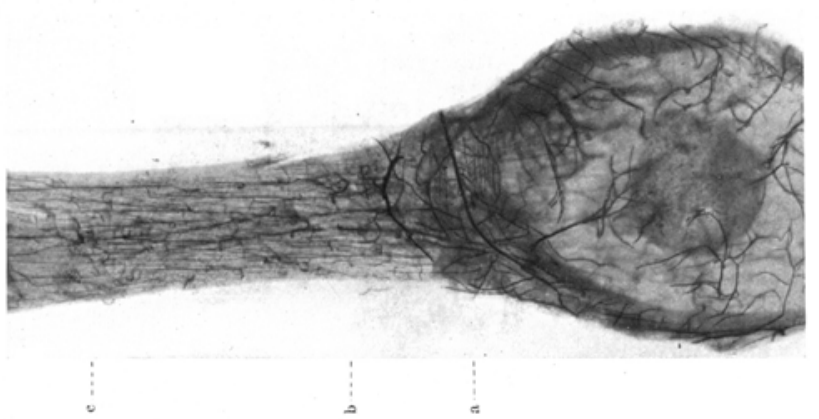

is

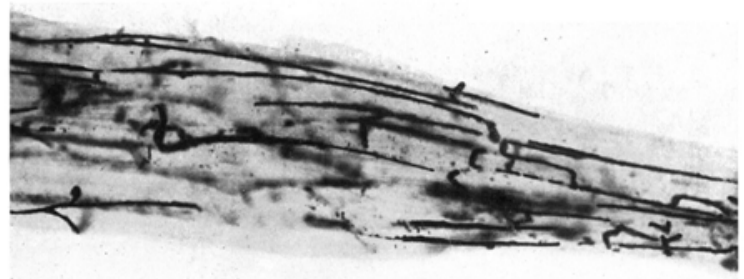

$+\dot{0}$

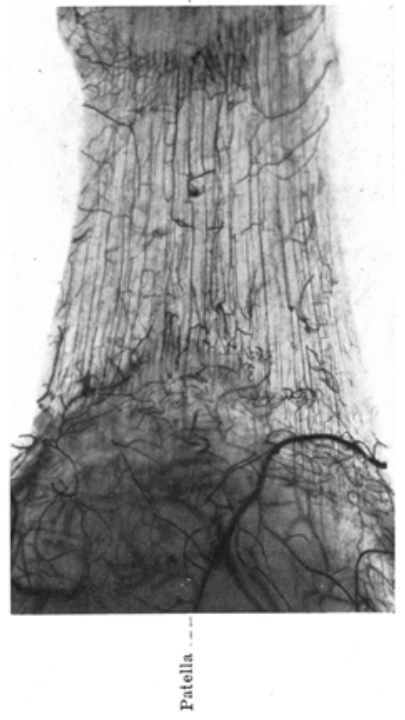

(i)
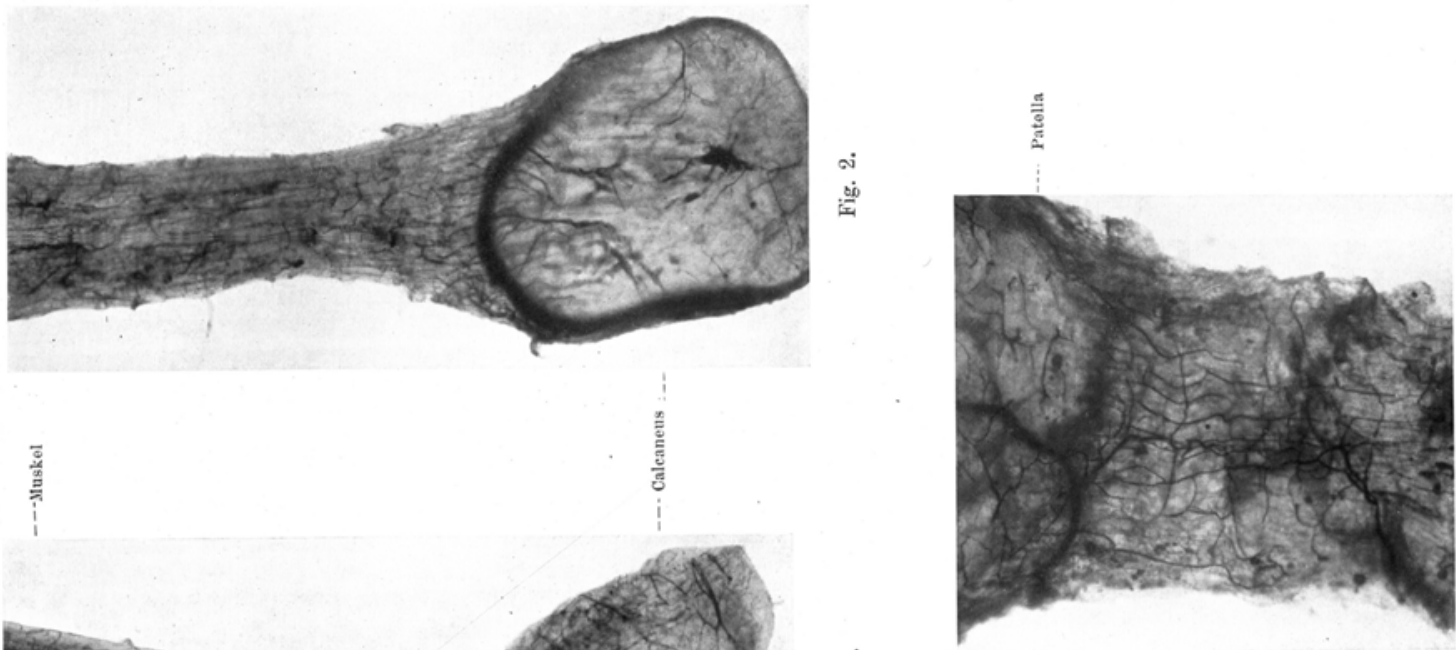

$\infty$

.60

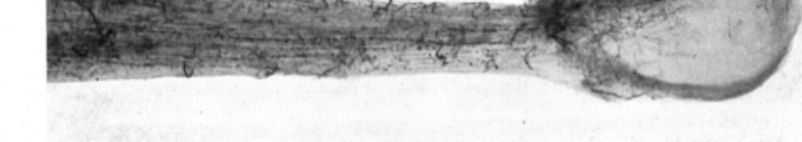




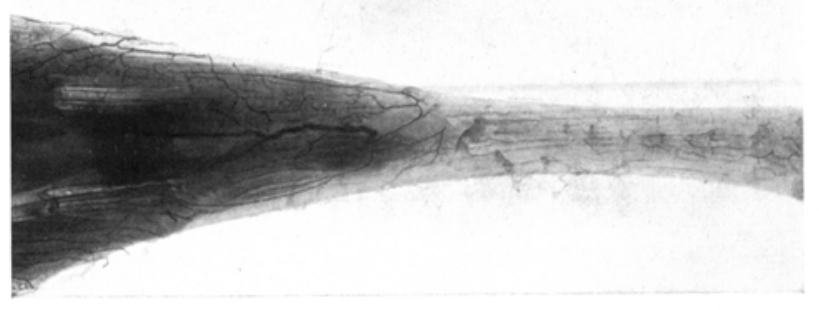

$\stackrel{1}{=}$

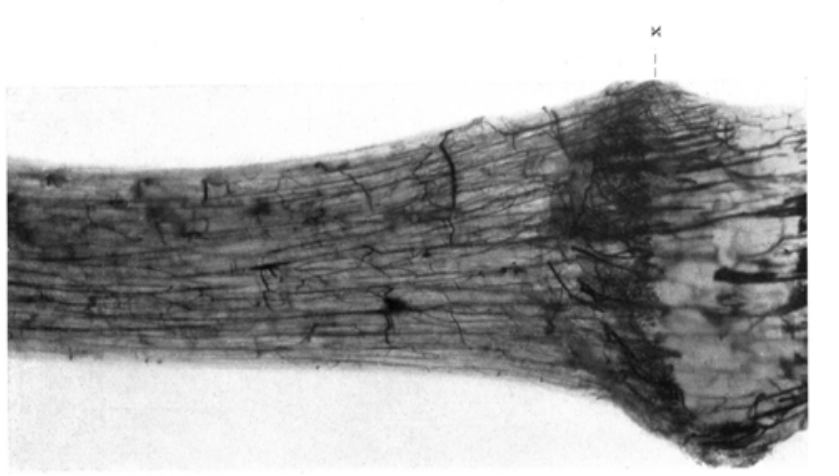

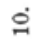

要

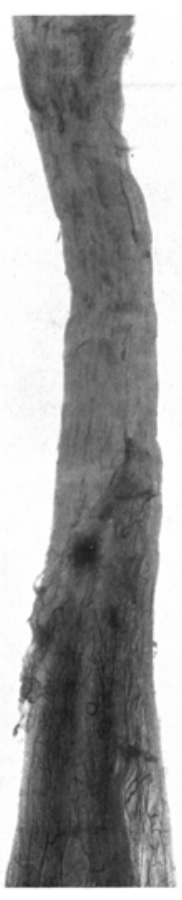

ำ
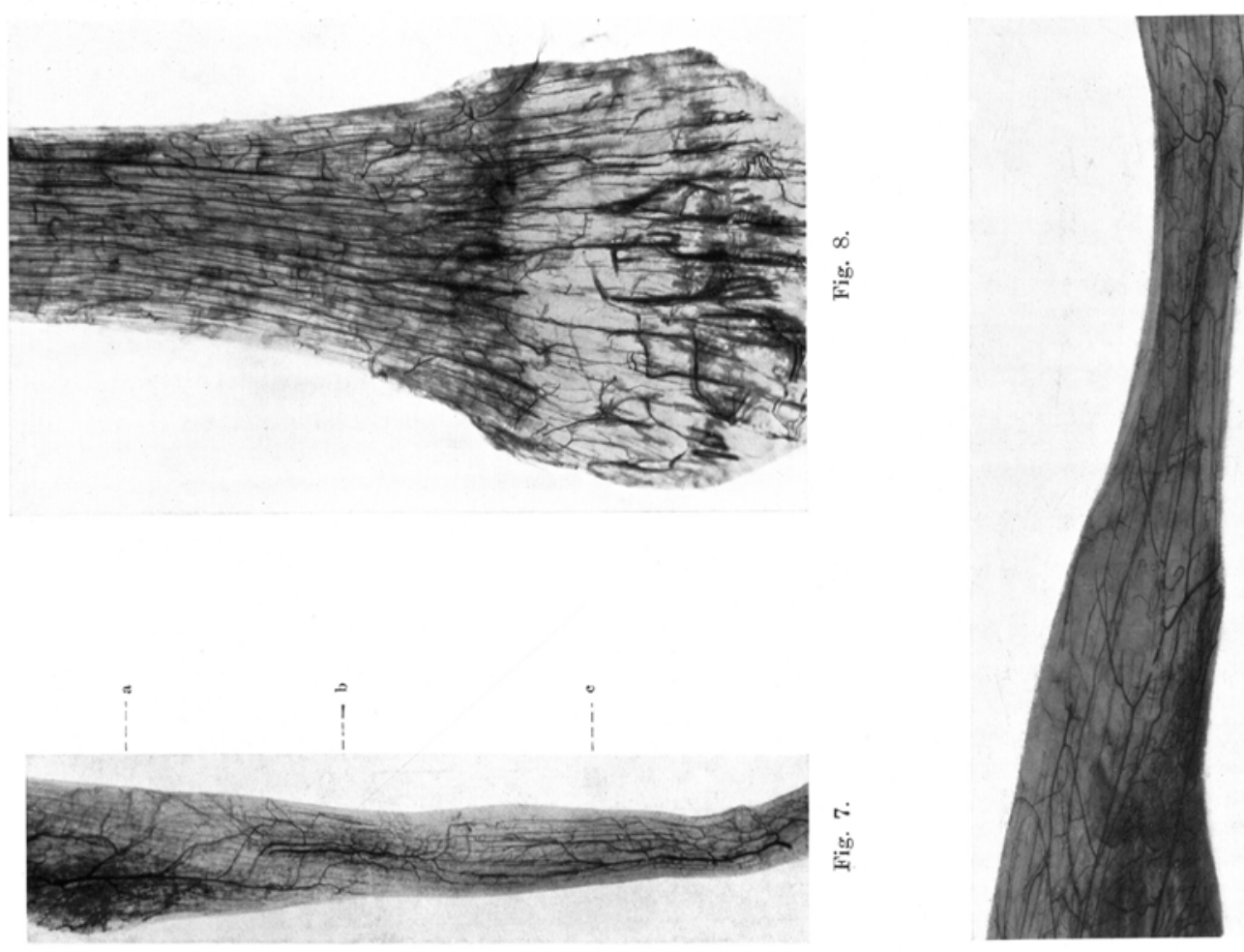

क 